\title{
Hypoglycemic and hypolipidemic effects of Nelumbo nucifera flower in Long-Evans rats
}

\author{
Dipa Islam $^{1 *}$, Amdadul Huque ${ }^{1}$, Sheuly ${ }^{2}$, Liton Chandra Mohanta ${ }^{1}$, Sadhan Kumar Das ${ }^{1}$, Abida Sultana ${ }^{3}$, Evena \\ Parvin Lipy ${ }^{1}$, U. K. Prodhan ${ }^{4}$
}

${ }^{1}$ Biomedical and Toxicological Research Institute (BTRI), Institute of Food Science and Technology, Bangladesh Council of Scientific and Industrial Research (BCSIR), Dhaka, Bangladesh

${ }^{2}$ Science, Secondary Education Quality and Access Enhancement Project (SEQAEP), Ministry of Education, Dhaka, Bangladesh

${ }^{3}$ Department of Food Technology, Kishoreganj Polytechnic Institute, Kishoreganj, Bangladesh

${ }^{4}$ Department of Food Technology and Nutritional Science, Mawlana Bhashani Science and Technology University, Bangladesh

\section{A R T I C L E I N F O}

Article Type:

Original Article

\section{Article History:}

Received: 15 September 2017

Accepted: 20 January 2018

Keywords:

Alloxan monohydrate

Diabetes

Hypoglycemia

Hypolipidemia

Medicinal plant

\begin{abstract}
A B S T R A C T
Introduction: The study was conducted to assess the hypolipidemic and hypoglycemic effects of Nelumbo nucifera flowers powder in Long-Evans rats.

Methods: Experimental rats were made hyperlipidemic and diabetic (type 2) by feeding high fat diet (Lab diet, Dalda and Coconut oil = 4: 3:1) and injecting alloxan respectively. $N$. nucifera flowers powder in different percentages was mixed with the regular Lab Diet for 21 days feeding. Serum total cholesterol, triglyceride, low-density lipoprotein (LDL), high-density lipoprotein (HDL) and blood glucose levels were evaluated in various groups.

Results: Feeding with $N$. nucifera flowers powder at different percentages to hyperlipidemic groups showed diverse but a significant $(P<0.05)$ decrease in serum total cholesterol, triglyceride and LDL-cholesterol levels when compared to control group while HDL-cholesterol level was increased significantly $(P<0.05)$. Routine feeding with $N$. nucifera flowers powder for 21 days resulted in significant decrease in the blood glucose levels of alloxan-induced diabetic rats. Both $20 \%$ and $10 \%$ of $N$. nucifera flower powders with Lab Diet significantly $(P<0.05)$ decreased blood glucose level up to $48 \%$ and $34 \%$, respectively in comparison to the drug control group treated with glibenclamide which was found with the decreasing capability up to $66 \%$ where the higher percentage of $N$. nucifera flower powder was found to exert more prominent effect in lowering blood glucose level. During comparison to the control group, the above mentioned percentages of $N$. nucifera flowers powder was found to reduce the blood glucose level around $32.05 \%$ and $47.92 \%$ respectively. Although not prominent, but the data revealed that the sample was endowed with the body weight declining capability.

Conclusion: Results of the experiments affirm that the flower of $N$. nucifera has potent hypoglycemic and hypolipidemic properties and might be useful in these patients.
\end{abstract}

Implication for health policy/practice/research/medical education:

Flowers of N. nucifera may be utilized for both diabetic and hyperlipidemic patients. Thus, further studies on it may reveal new active compounds having anti-diabetic and lipid lowering properties.

Please cite this paper as: Islam D, Huque A, Sheuly, Mohanta LC, Das SK, Sultana A, Lipy EP, Prodhan UK. Hypoglycemic and hypolipidemic effects of Nelumbo nucifera flower in Long-Evans rats. J Herbmed Pharmacol. 2018;7(3):148-154. doi: 10.15171/ jhp.2018.25.

\section{Introduction}

Lipid abnormalities are generally connected with diabetes which ultimately is considered as one of the most contributory factors for cardiovascular morbidity and mortality in patients suffering from diabetes. Type II diabetes is the most common one which accounts for about $90 \%$ of the diabetic population affecting minimum
15 million people. It is directly connected with other difficulties including hypertension, atherosclerosis and microcirculatory disorders. Among all the endocrine disorders, diabetes mellitus (type 2) is highly reported and is projected that almost three hundred million will be affected by the disease by 2025 and greater portion of diabetic population will be from India, China and 
United States (1-6). According to recent epidemiological studies, in Bangladesh more than 11\% diabetic patients are suffered from type II diabetes and the presence of atypical fasting glucose, which triggers the initiation of diabetes, goes beyond 6\% in Dhaka city (7). Therefore, it is now inevitable to search for novel oral drugs that will be therapeutically effective both as hypoglycemic and hypolipidemic agents at affordable cost particularly both for the developing and under-developed countries. In the experiments of hypoglycemic effects evaluation, animals are made diabetic usually by injecting alloxan or streptozotocin (STZ) intraperitoneally (IP) or intravenously (IV). For the studies of hypolipidemic activity, the subjected animals are generally fed with high fat diet to produce hyperlipidemia in those animals. Nelumbo nucifera is an aquatic perennial which belongs to the family of Nelumbonaceae and is familiar by many local names (e.g. Indian lotus, Chinese water lily, and Sacred lotus). Lotus flowers powder is utilized in traditional medicine to treat high blood pressure, diarrhea, fever, weakness, infection, skin inflammation, and body heat imbalance (8). It is also an effective treatment against abnormal bleeding such as hematemesis, epistaxis, hemoptysis, hematuria, and metrorrhagia (9). Lotus flowers extracts are found to possess a powerful antioxidant and radical scavenging ability along with inhibitory activity of diabetic complications (10-13). Lotus flower extract has been reported to treat obesity, too (1416). Furthermore, lotus flowers extracts were observed to modulate lipolysis-activity and decrease adipogenesis in human pre-adipocytes (17) as well as to increase cholesterol profile in mice and reduce levels of phospholipids and triglycerides (18). It is notable to mention that anti-HIV principles have also been constructed using the ethanolic extract of the lotus flowers (19). This study was conducted to assess the hypolipidemic and hypoglycemic effects of N. nucifera flowers powder in Long-Evans rats.

\section{Materials and Methods}

\section{Experimental design}

This experiment was conducted according to Figure 1. The study was carried out in Biomedical and Toxicological Research Institute (BTRI), Bangladesh Council for Scientific and Industrial Research (BCSIR), Dhaka, Bangladesh. Flowers of N. nucifera were brought from National Botanical Garden, Mirpur, Dhaka, Bangladesh and identified by the Bangladesh National Herbarium, Dhaka. At first, the flowers of $N$. nucifera were washed vigorously using water and then dried at room temperature. The flowers were then air dried and subsequently oven dried at $37^{\circ} \mathrm{C}$ temperature. Later on, the dried flowers were grinded to powder form which then screened to find out the fine powder. Powder size of $<0.5 \mathrm{~mm}$ after passing through a 35-mesh sieve was considered for the experiment.

Healthy male rats (Long-Evans) of local strain, having around 150-200 $\mathrm{g}$ and 200-250 g weights were taken for the hypolipidemic and hypoglycemic effects studies, respectively. The proper environmental condition for the experimental rats was confirmed, kept under firm supervision for a week and maintained at a constant room temperature of $25^{\circ} \mathrm{C} \pm 5^{\circ} \mathrm{C}$ with humidity of $40 \%$ to $70 \%$

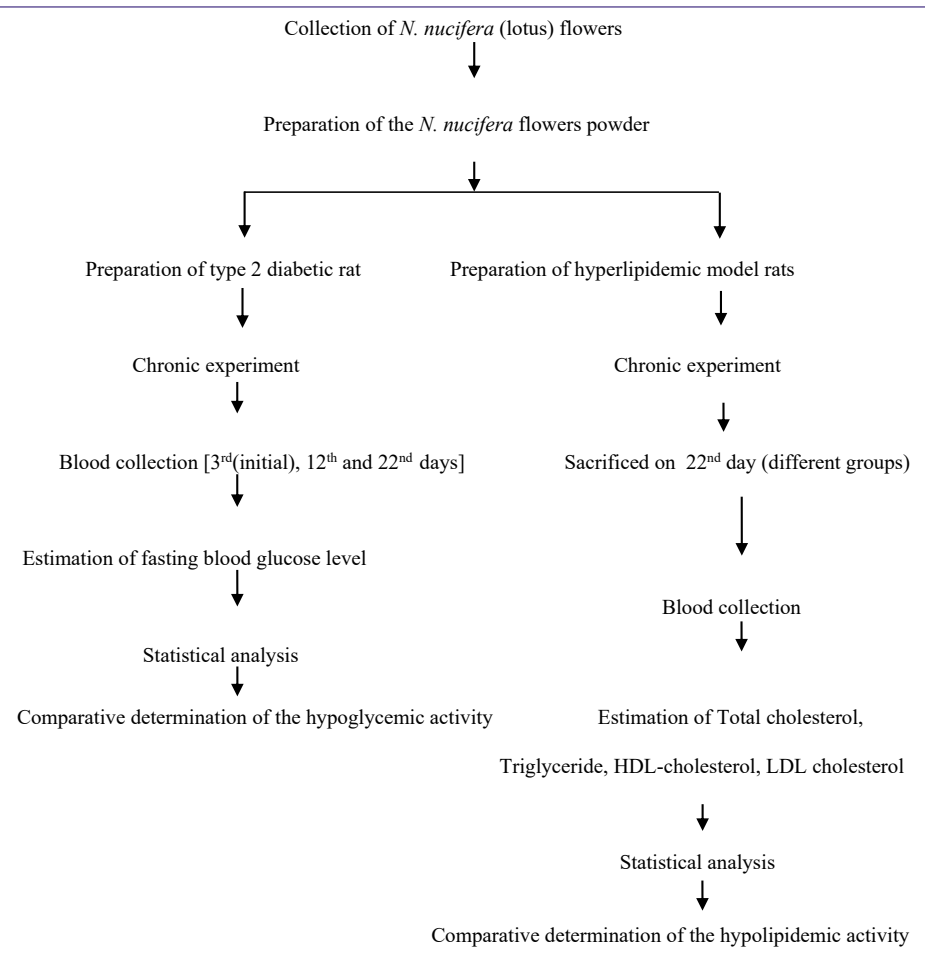

Figure 1. Experimental design 
with natural day-night cycle.

For the hypolipidemic effect studies, the rats were segregated into a total of eight groups consisting of six in each maintaining the congruity of body weight. These included:

Group-A (feeding with lab diet).

Group-B1 (feeding with $10 \%$ powder of N. nucifera flower and $90 \%$ lab diet).

Group-B2 (feeding with 20\% powder of N. nucifera flower and $80 \%$ lab diet).

Group-B3 (feeding with 30\% powder of $N$. nucifera flower and $70 \%$ lab diet).

Group-C (feeding with high fat diet after inducing hyperlipidemia).

Group-D1 (feeding with $10 \%$ powder of N. nucifera flower and $90 \%$ high fat diet after inducing hyperlipidemia).

Group-D2 (feeding with 20\% powder of N. nucifera flower and $80 \%$ high fat diet after inducing hyperlipidemia).

Group-D3 (feeding with 30\% powder of N. nucifera flower and $70 \%$ high fat diet after inducing hyperlipidemia).

For evaluation of hypoglycemic activity of $N$. nucifera flower, the rats were segregated into five groups comprising of six in each with the congruence of body weight. These included:

1) Group-A (Normal Control, rats fed with $100 \%$ lab diet)

2) Group-B (Alloxan induced diabetic rats fed with $100 \%$ lab diet, diabetic control).

3) Group-C (Alloxan induced diabetic rats fed with Lab Diet plus glibenclamide, given at a dose of $5 \mathrm{mg} / 10 \mathrm{~mL}$ (9.9 $\mathrm{mL} \mathrm{H} \mathrm{H}_{2} \mathrm{O}+0.1 \mathrm{~mL}$ Twin 20$) / \mathrm{kg}$ body weight, drug control (23).

4) Group-D (Alloxan induced diabetic rats fed with 10\% powder of $N$. nucifera flower plus $90 \%$ lab diet).

5) Group-E (Alloxan induced diabetic rats fed with $20 \%$ powder of $N$. nucifera flower plus $80 \%$ lab diet).

Before starting the experiment, the weights of the rats were taken precisely and the rats were marked on the tail, right front, right back, left front, left back and kept unmark which was subsequently applied as identification purpose for a particular rat, so that the reaction of a specific rat before and after the drug administration could be observed individually.

Preparation of hyperlipidemic rats and estimation of the lipid profile

The rats were made hyperlipidemic by feeding high fat diet for 10 days containing Lab Diet, Dalda and Coconut oil in 4:3:1 ratio.

For evaluation of hypolipidemic activity, the powder of the $N$. nucifera flower was administered orally with high fat diet and lab diet at doses of daily 10\%, 20\% and 30\% of the regular diet for 21 days.

Blood samples were collected on the 22nd day by sacrificing the rats after making anesthesia using ketamine hydrochloride. After sacrificing about $2 \mathrm{~mL}$ of blood was taken cautiously. The blood samples were then centrifuged after 20 minutes at $4000 \mathrm{rpm}$ for 10 minutes and recentrifuged at $2000 \mathrm{rpm}$ for 5 minutes. After that the serums samples were separated and taken into Eppendorf tubes. Then the serum triglyceride, total cholesterol, highdensity lipoprotein cholesterol (HDL-C) and low-density lipoprotein cholesterol (LDL-C) were measured. One milliliter of serum was aliquoted and kept frozen at $-20^{\circ} \mathrm{C}$ until analysis of serum for lipid profile.

Serum total cholesterol was measured by enzymatic colorimetric (Cholesterol Oxidase /Peroxidase, CHODPAP) method (Randox Laboratories Ltd., UK) using autoanalyzer, AutoLab. Serum HDL-cholesterol was estimated by enzymatic colorimetric (Cholesterol CHODPAP) method (Randox Laboratories Ltd., UK) using microplate reader (Bio-Tec, ELISA) and Serum triglyceride (TG) was examined by enzymatic colorimetric (GPOPAP) method (Randox Laboratories Ltd., UK) using auto analyzer, Auto Lab. Then, serum LDL cholesterol was calculated manually. The calculated formula was:

$$
L D L-C=T C-\left(\frac{T G}{5}+H D L-C\right)
$$

All the groups of rats were remained under similar environmental conditions and provided with the measured food and water throughout the experiment. The body weight of each rat was measured and compared with the controls.

Preparation of diabetic rats and measurement of blood glucose level

Alloxan monohydrate $\left(\mathrm{C}_{2} \mathrm{H}_{2} \mathrm{~N}_{2} \mathrm{O}_{4} \cdot \mathrm{H}_{2} \mathrm{O}\right)$ was available in colored bottles containing $25 \mathrm{~g}$ powder. The solution was prepared by dissolving $10 \mathrm{~g}$ in $100 \mathrm{~mL}$ of distilled water $(10 \%)$.

The rats were made diabetic (diabetes mellitus) by injecting alloxan monohydrate $150-\mathrm{mg} / \mathrm{kg}$-body weight intravenously (20). Three days after injection of the alloxan monohydrate, blood glucose of all the surviving rats was determined by the Diagnostics EliTech method. The rats with blood glucose levels above $6 \mathrm{mmol} / \mathrm{L}$ were considered as diabetic and considered for further study. For the assessment of hypoglycemic effect, N. nucifera flowers powder was fed orally with Lab Diet at doses of daily $10 \%$ and $20 \%$ of regular diet for 21 days. The drug, glibenclamide was administrated orally as a drug control, at a dose of $5 \mathrm{mg} / 10 \mathrm{~mL}\left(9.9 \mathrm{~mL} \mathrm{H}_{2} \mathrm{O}+0.1 \mathrm{ml}\right.$ Twin 20)/ $\mathrm{kg}$ body weight to the respective grouped rats.

Fasting blood glucose level was measured using glucometer on the 3rd (initial), 12th and 22nd days by collecting blood samples nicking the lateral tail vein using a sterile scalpel blade under ketamine hydrochloride anesthesia. Just before cutting, the tail was immersed into warm water $\left(40^{\circ} \mathrm{C}\right)$ for around 25 seconds for vasodilatation.

Data analysis

The data analysis was performed using SPSS. 11.5 
windows program. $P<0.05$ was considered significant. For charts and graphical representation Microsoft Word and Microsoft Excel were used.

\section{Results}

During the entire study period laboratory protocols described in Materials and Methods section were followed strictly. The findings based on experimental data are depicted below:

Chronic effect of Nelumbo nucifera flower powder on body weight of rats

Chronic effect of $N$. nucifera flower powder on body weight of normal control rats fed with lab diet and rats fed with lab diet plus sample

The impacts of $N$. nucifera flowers powder on body weight of the rats during 21 days of feeding with Lab Diet plus sample in different percentages are shown in Table 1. Body weight of each rat was measured at every 11-day intervals and compared with that of the normal control group fed with $100 \%$ Lab Diet.

Chronic effect of Nelumbo nucifera flower powder on body weight of hyperlipidemic model rats fed with high fat diet plus sample in comparison to hyperlipidemic model rats fed with only high fat diet

The impacts of $N$. nucifera flower powder on body weight of hyperlipidemic rats administered with 100\% high fat diet and the grouped rats fed with high fat diet plus sample at various percentages during 21 days of feeding are delineated in Table 2. Body weight of each rat was measured at every 11-day intervals.
Chronic effects of Nelumbo nucifera flower powder on lipid status (total cholesterol \& triglyceride) of rats Chronic effects of Nelumbo nucifera flower powder on lipidemic status (total cholesterol \& triglyceride) of rats fed with Lab Diet plus sample in comparison to normal control rats fed with lab diet

The mean serum total cholesterol and triglyceride levels of normal control rats administered with lab diet and model rats, administered with Lab Diet plus $N$. nucifera flowers powder for 21 days followed by blood collection on 22nd day are presented in Table 3. To find out whether there was a statistically significant difference in hypolipidemia obtained by the powder sample on the 22 nd day.

Chronic effect of Nelumbo nucifera flowers powder on lipidemic status (total cholesterol \& triglyceride) of hyperlipidemic rats fed with high fat diet Plus Sample in comparison to hyperlipidemic model rats fed with only high fat diet

The mean serum total cholesterol and triglyceride levels of high fat diet treated hyperlipidemic rats administered with only high fat diet (treated as control) and model rats administered with high fat diet plus powder of $N$. nucifera flower for 21 days followed by blood collection on 22nd day are presented in Table 4.

Chronic effect of Nelumbo nucifera flower powder on lipidemic status (HDL-C and LDL-C) of rats Chronic effect of Nelumbo nucifera flower powder on lipidemic status (HDL-C and LDL-V) of rats fed with lab diet plus sample in comparison to normal control rats fed with lab diet

The effect of N. nucifera flower powder on atherogenic

Table 1. Chronic effect of Nelumbo nucifera flower powder on body weight (BW) of rats fed with lab diet plus sample in comparison to normal control rats fed with lab diet

\begin{tabular}{lllll}
\hline Group & & BW, Initial day (g) & BW, 12th day (g) & BW, 22nd day (g) \\
\hline Group A (100\% high diet) $(\mathrm{n}=6)$ & $174.17 \pm 8.04(100 \%)$ & $178.17 \pm 7.78(102.30 \%)$ & $183.17 \pm 7.31(105.18 \%)$ \\
Group B (lab diet + sample) & B1 (90\% lab diet + 10\% sample) $(\mathrm{n}=6)$ & $172 \pm 7.04(100 \%)$ & $173.83 \pm 7.68^{\mathrm{a}}(101.06 \%)$ & $176 \pm 7.82^{\mathrm{a}}(102.33 \%)$ \\
& B2 $(80 \%$ lab diet $+20 \%$ sample) $(\mathrm{n}=6)$ & $171.17 \pm 6.43(100 \%)$ & $172.83 \pm 6.11^{\mathrm{a}}(100.97 \%)^{\mathrm{a}}$ & $174.83 \pm 6.11^{\mathrm{a}}(102.12 \%)$ \\
& B3 $(70 \%$ lab diet $+30 \%$ sample) $(\mathrm{n}=6)$ & $172.67 \pm 6.19(100 \%)$ & $174.50 \pm 6.09^{\mathrm{a}}(101.06 \%)$ & $174.83 \pm 5.67^{\mathrm{a}}(101.25 \%)$ \\
\hline
\end{tabular}

Data are presented as Mean \pm SD and compared using one-way ANOVA (Duncan post hoc test), $\mathrm{n}=$ number of rats.

a Statistically significant in comparison to the control group $(P<0.05)$.

Table 2. Chronic effect of Nelumbo nucifera flower powder on body weight (BW) of hyperlipidemic rats fed with high fat diet plus sample in comparison to hyperlipidemic rats fed with only high fat diet

\begin{tabular}{|c|c|c|c|c|}
\hline Group & & BW, Initial day (g) & BW, 12th day (g) & BW, 22nd day (g) \\
\hline Group C (100\% high fat diet $(n=6)$ & & $172.33 \pm 10.46$ & $176.83 \pm 10.52$ & $180.33 \pm 9.63$ \\
\hline \multirow[t]{3}{*}{ Group D (high fat diet + sample) } & B1 (90\% lab diet $+10 \%$ sample) $(n=6)$ & $171.67 \pm 11.00$ & $172.20 \pm 11.62^{\mathrm{a}}$ & $172.14 \pm 12.58^{a}$ \\
\hline & B2 (80\% lab diet $+20 \%$ sample) $(n=6)$ & $171.50 \pm 8.17$ & $172.58 \pm 7.06^{\mathrm{a}}$ & $172.59 \pm 7.17^{\mathrm{a}}$ \\
\hline & B3 (70\% lab diet $+30 \%$ sample) $(n=6)$ & $174 \pm 5.93$ & $174.88 \pm 5.47^{a}$ & $174.79 \pm 5.92^{\text {a }}$ \\
\hline
\end{tabular}

Data are presented as Mean \pm SD and compared using one-way ANOVA (Duncan post hoc test), $\mathrm{n}=$ number of rats.

a Statistically significant in comparison to the control group $(P<0.05)$. 
Table 3. Chronic effects of Nelumbo nucifera flower powder on lipidemic status (total cholesterol \& triglyceride) of rats fed with lab diet plus sample in comparison to normal control rats fed with lab diet

\begin{tabular}{|c|c|c|c|c|}
\hline \multicolumn{2}{|l|}{ Group } & BW, Initial day (g) & BW, 12th day (g) & BW, 22nd day (g) \\
\hline \multicolumn{2}{|c|}{ Group C (100\% high fat diet) $(n=6)$} & $172.33 \pm 10.46(100 \%)$ & $176.83 \pm 10.52(102.61 \%)$ & $180.33 \pm 9.63(104.64 \%)$ \\
\hline \multirow{3}{*}{$\begin{array}{l}\text { Group D (high fat } \\
\text { diet + sample) }\end{array}$} & D1 (90\% high fat diet $+10 \%$ sample) $(n=6)$ & $171.67 \pm 11.00(100 \%)$ & $172.20 \pm 11.62^{\mathrm{a}}(100.31 \%)$ & $172.14 \pm 12.58^{\mathrm{a}}(100.27 \%)$ \\
\hline & D2 ( $80 \%$ high fat diet $+20 \%$ sample) $(n=6)$ & $171.50 \pm 8.17(100 \%)$ & $172.58 \pm 7.06^{\mathrm{a}}(100.58 \%)$ & $172.59 \pm 7.17^{\mathrm{a}}(100.64 \%)$ \\
\hline & D3 (70\% high fat diet $+30 \%$ sample $(n=6)$ & $174 \pm 5.93(100 \%)$ & $174.88 \pm 5.47^{\mathrm{a}}(100.51 \%)$ & $174.79 \pm 5.92^{\mathrm{a}}(100.45 \%)$ \\
\hline
\end{tabular}

Data are presented as Mean \pm SD and compared using one-way ANOVA (Duncan post hoc test), $\mathrm{n}=$ number of rats.

a Statistically significant in comparison to the control group $(P<0.05)$.

Table 4. Chronic effect of Nelumbo nucifera flowers powder on lipidemic status (total cholesterol \& triglyceride) of hyperlipidemic rats fed with high fat diet plus sample in comparison to hyperlipidemic model rats fed with only high fat diet

\begin{tabular}{|c|c|c|c|}
\hline Group & & TC, 22nd day (mg/dL) & TG, 22nd day (mg/dL) \\
\hline \multirow[t]{2}{*}{ Group C (100\% high fat diet) $(n=6)$} & & $119.33 \pm 11.13$ & $100.83 \pm 18.49$ \\
\hline & D1 (90\% high fat diet $+10 \%$ sample $)(n=6)$ & $85.17 \pm 9.26^{a}$ & $89.83 \pm 16.39^{a}$ \\
\hline \multirow[t]{2}{*}{ Group D (high fat diet + sample) } & D2 (80\% high fat diet $+20 \%$ sample $)(n=6)$ & $72.67 \pm 8.14^{a}$ & $83 \pm 15.28^{a}$ \\
\hline & D3 $(70 \%$ high fat diet $+30 \%$ sample $)(n=6)$ & $60.17 \pm 8.18^{a}$ & $77.83 \pm 14.16^{\mathrm{a}}$ \\
\hline
\end{tabular}

Data are presented as mean \pm SD and compared using one-way ANOVA (Duncan post hoc test), $\mathrm{n}=$ number of rats.

a Statistically significant in comparison to the control group $(P<0.05)$.

lipids (HDL-C and LDL-V) of rats fed with lab diet plus sample and normal control rats fed with lab diet is shown in Table 5.

Chronic effect of Nelumbo nucifera flowers powder on lipidemic status (HDL-C and $L D L-V$ ) of high fat diet induced hyperlipidemic rats fed with high fat diet plus sample in comparison to hyperlipidemic rats fed with only high fat diet

The effect of $N$. nucifera flower powder on atherogenic lipids (HDL-C and LDL-V) is presented in Table 6.

Chronic effect of Nelumbo nucifera flowers powder on fasting blood glucose concentration of alloxan induced type 2 diabetic model rats

The effects of N. nucifera flower powder $10 \%$ with $90 \%$ Lab Diet and 20\% with 80\% Lab Diet, expressed as change in blood glucose level, are depicted in Table 7. Fasting blood glucose level of each rat was taken at every 11-day intervals.

\section{Discussion}

It is obvious that there was a tendency to decline the body weight in all sample fed rat groups whereas normal control rats displayed increase in body weights at the end of study period (Table 1). Mathematically around a maximum $0.90 \%$ body weight reduction was observed in comparison to the body weights measured at first day considered as $100 \%$.

It is noticed from the data presented in Table 2 that there was a raise in body weight in each group. But the sample administered groups displayed slower enhancement in body weight than high fat diet fed groups. Data also suggest that after feeding of only high fat diet for 21 days, body weight augmentation was almost maximum of $10 \%$ if the initial body weight was considered as $100 \%$. But, when along with the high fat diet, the sample at different percentages was given, the body weight increase was only a maximum of $0.20 \%$ considering the initial body weight as $100 \%$.

According to data presented in Table 3, a significant diminution $(P<0.05)$ of TC and TG values in group B1 ( $90 \%$ lab diet $+10 \%$ sample), group B2 (80\% lab diet + $20 \%$ sample) and group B3 (70\% lab diet $+30 \%$ sample) compared to group A (100\% Lab Diet) was observed and this diminution was more significant in higher percent of

Table 5. Chronic effect of Nelumbo nucifera flower powder on lipidemic status (HDL-C and LDL-C) of rats fed with lab diet plus sample in comparison to normal control rats fed with lab diet

\begin{tabular}{llcc}
\hline Group & & HDL-C, 22 ${ }^{\text {nd }}$ day & LDL-C, 22 ${ }^{\text {nd }}$ day \\
\hline Group A (100\% lab diet) $(n=6)$ & & $55.50 \pm 4.42$ & $16.85 \pm 2.44$ \\
& B1 (90\% lab diet + 10\% sample) $(n=6)$ & $69.83 \pm 7.91^{\text {a }}$ & $9.00 \pm 1.21^{\text {a }}$ \\
Group B (lab diet + sample) & B2 (80\% lab diet + 20\% sample) $(n=6)$ & $76 \pm 9.78^{\text {a }}$ & $8.33 \pm 1.08^{\text {a }}$ \\
& B3 (70\% Lab diet + 30\% sample) $(n=6)$ & $80.83 \pm 9.70^{\text {a }}$ & $7.28 \pm 1.01^{\text {a }}$ \\
\hline
\end{tabular}

Data are presented as mean \pm SD and compared using one-way ANOVA (Duncan post hoc test), $n=$ number of rats.

${ }^{a}$ Statistically significant in comparison to the control group $(P<0.05)$. 
Table 6. Chronic effect of Nelumbo nucifera flower powder on lipidemic status (HDL-C and LDL-C) of high fat diet induced hyperlipidemic model rats fed with high fat diet plus sample in comparison to hyperlipidemic model rats fed with only high fat diet

\begin{tabular}{|c|c|c|c|}
\hline Group & & HDLC, 22nd day & LDLC, 22nd day \\
\hline \multirow[t]{2}{*}{ Group C (100\% high fat diet) $(n=6)$} & & $50.67 \pm 2.42$ & $21.20 \pm 4.53$ \\
\hline & D1 (90\% high fat diet $+10 \%$ sample) $(n=6)$ & $75 \pm 7.46^{a}$ & $14.03 \pm 2.54^{\mathrm{a}}$ \\
\hline \multirow[t]{2}{*}{ Group D (high fat diet + sample) } & D2 (80\% high fat diet $+20 \%$ sample) $(n=6)$ & $79 \pm 6.90^{a}$ & $12 \pm 1.92^{\mathrm{a}}$ \\
\hline & D3 (70\% high fat diet $+30 \%$ sample) $(n=6)$ & $89.17 \pm 5.42^{\mathrm{a}}$ & $9.60 \pm 1.16^{\mathrm{a}}$ \\
\hline
\end{tabular}

Data are presented as mean \pm SD and compared using one-way ANOVA (Duncan post hoc test), $\mathrm{n}=$ number of rats.

a Statistically significant in comparison to the control group $(P<0.05)$.

Table 7. Chronic effect of Nelumbo nucifera flower powder on fasting blood glucose (FBG) concentration of alloxan induced type 2 diabetic model rats

\begin{tabular}{llll}
\hline Group & FBG, initial day (mmol/L) & FBG, 12th day (mmol/L) & FBG, 22nd day (mmol/L) \\
\hline Group-A (normal control) & $4.80 \pm 0.51(100 \%)$ & $4.76 \pm 0.43(99.17 \%)$ & $4.74 \pm 0.39(98.75 \%)$ \\
Group-B (diabetic control) & $15.42 \pm 0.23(100 \%)$ & $15.38 \pm 0.18(99.74 \%)$ & $15.64 \pm 0.25(101.43 \%)$ \\
Group-C (drug control) & $15.50 \pm 0.28(100 \%)$ & $9.41 \pm 0.38^{\mathrm{a}}(60.71 \%)$ & $5.32 \pm 0.16^{\mathrm{a}}(34.32 \%)$ \\
Group-D (sample 10\% + lab diet 90\%) & $15.07 \pm 0.47(100 \%)$ & $11.49 \pm 0.87^{\mathrm{a}}(76.24 \%)$ & $10.24 \pm 0.96{ }^{\mathrm{a}}(67.95 \%)$ \\
Group-E (sample 20\% + lab diet 80\%) & $15.13 \pm 0.58(100 \%)$ & $9.76 \pm 0.57^{\mathrm{a}}(64.51 \%)$ & $7.88 \pm 0.33{ }^{\mathrm{a}}(52.08 \%)$ \\
\hline
\end{tabular}

Data are presented as mean \pm SD and compared using one-way ANOVA (Duncan post hoc test), $\mathrm{n}=$ number of rats.

a Statistically significant in comparison to the control group $(P<0.05)$.

Sample treated groups. Data reveal that the TC and TG values of the highest percentage sample treated group, group B3 in comparison to the group A after 21 days of feeding, were found to be lowered by around $43 \%$ and $44 \%$, respectively.

Data shown in Table 4, confirm that samples have the characteristics of TC and TG diminishing abilities at a significant level in comparison to that of control one. In Table 5, significant variations were observed in the HDL-C and LDL-C levels among all the test groups after 21 days of sample treatment. In case of group B3 (70\% lab diet $+30 \%$ sample) HDL-cholesterol was mostly augmented compared to the group A (100\% lab diet). In Groups B1 (90\% lab diet + 10\% sample) and B2 (80\% lab diet $+20 \%$ sample) the HDL-C levels were also found to be augmented in a significant level although lower than group B3. Furthermore, LDL-C level was observed to be reduced significantly in all the studies groups. Among all the groups, group B3 (70\% lab diet $+30 \%$ sample) was found with the lowest level of LDL-C. In group B3, the HDL-C level was observed to be augmented by almost $49 \%$ and the LDL-C level was found to be reduced to $60 \%$ in comparison to that of group A after 21 days of sample treatment.

It was found according to data presented in Table 6 that, overall, HDL-C level increased significantly while that of LDL-C decreased which was also significant among all the studied groups after 21 days of chronic trial. The lipidemic profile was checked on 22 nd day of the study. In case of group D3 (70\% lab diet + 30\% sample) HDL-C level was found to be increased maximum compared to the group C (100\% lab diet). It is worth mentioning that in case of group D1 ( $90 \%$ lab diet $+10 \%$ sample) and group D2 (80\% lab diet + 20\% sample) the HDL-C level was also observed to be augmented significantly while LDL-C level in all experimental groups was found to be decreased significantly. More specifically, in case of group D3 where the maximum of around $48 \%$ of HDL-C level was observed to be increased and the highest of $62 \%$ of LDL-C level seen to be reduced in comparison to that of group $\mathrm{C}$.

It is worth mentioning from Table 5 and Table 6 that the increase of HDL cholesterol level was prominently higher in sample diet fed groups compared to that of normal control groups (both group A and group C). Significantly increment tendency of HDL cholesterol level was observed with the higher percentage of sample diet. It is also clear from Table 5 and Table 6 that the reduction of LDL cholesterol level was higher with the higher percentage of sample diet which surely implies to the hypolipidemic activities of the sample, $N$. nucifera flowers powder.

In Table 7, blood glucose levels were depicted and more significant $(P<0.05)$ anti-diabetic activity was observed on 22 d day in alloxan induced type 2 diabetic model rats. In a study it has been suggested that a $25 \%$ reduction in blood glucose level is considered a significant hypoglycemic effect. The results of the study were satisfactory and revealed that the $10 \% \& 20 \% \mathrm{~N}$. nucifera flowers powder exhibited significant $(P<0.05)$ hypoglycemic activity. In case of $10 \%$ sample induced group, the reduction of blood glucose level was 33\%. The reduction of blood glucose level in alloxan induced rat was found highest, $44 \%$ with the N. nucifera of $20 \%$.

\section{Conclusion}

According to the experimental results, it can be affirmed that $N$. nucifera flowers powder is endowed with strong hypoglycemic and hypolipidemic properties. Therefore, powder of $N$. nucifera flower may be utilized for the management of diabetes mellitus and other related complications directly linked to the lipid disorders. On the whole, $N$. nucifera is a very promising herbal plant 
having enormous medicinal values. The plant should be examined vigorously to find out for its active ingredients for which hypoglycemic and hypolipidemic activities are being exerted and mode of actions behind the observed effects.

\section{Acknowledgements}

We are expressing our heartfelt gratitude to the Biomedical and Toxicological Research Institute

(BTRI), IFST, BCSIR, Dhaka, Bangladesh, for protruding the required hands during the study in all respects including financial and logistic supports.

\section{Authors' contribution}

DI generated the idea. AH, S, LCM, SKD, AS and EPL executed the studies in accordance with the designed procedure working in the laboratories and capturing data. DI, AH and UKP performed data analysis. S and AS prepared the initial draft. AH revised the draft and produced the final manuscript with the consent of DI. All are well concerned about the latest version and agreed for publication.

\section{Conflict of interests}

The authors of the manuscript affirm that they have no conflict of interest.

\section{Ethical considerations}

The research protocol and all the tests performed in this study were approved by the Animal Care Committee of BCSIR (No. 39.307.099.00.00.166.2010-455). All the associated ethical matters were considered to the best by the authors.

\section{Funding/Support}

Financial and logistic supports were provided by Biomedical and Toxicological Research Institute (BTRI), (BTRI), IFST, BCSIR, Dhaka, Bangladesh, Tel: +880 2 58610717.

\section{References}

1. Ramachandran A, Snehalatha C, Ma RC. Diabetes in South-East Asia: an update. Diabetes Res Clin Pract. 2014;103(2):231-7.

2. Kesari AN, Kesari S, Singh SK, Gupta RK, Watal G. Studies on the glycemic and lipidemic effect of Murraya koenigii in experimental animals. J Ethnopharmacol. 2007;112:305-11.

3. Edem DO. Hypoglycemic effects of ethanolic extracts of alligator pear seed (Persea americana Mill) in rats. Eur J Sci Res. 2009;33:669-678.

4. Bastaki S. Review diabetes mellitus and Its treatment. Int J Diab Met. 2005;13:111-34.

5. King H, Auburt RE, Herman WH. Global burden of diabetes 1995-2025: prevalence, numerical estimates and projections. Diab Care. 1998;21:1414-1431. doi: 10.2337/ diacare.21.9.1414.

6. Ramachandran A, Snehalatha C, Kapur A, Vijay V, Mohan
V, Das AK, et al. High prevalence of diabetes and impaired glucose tolerance in India: National Urban Diabetes Survey.. Diabetologia. 2001;44:1094-100. Doi: 10.1007/ s001250100627.

7. Sayeed MA, Rumi MAK, Ali L. Effect of socioeconomic risk factors on the difference in prevalence of diabetes between rural and urban populations in Bangladesh. Biol Med Res Council. 2007;33:1-12.

8. Sridhar KR, Rajeev B. Lotus-a potential nutraceutical source. J Agr Technol. 2007;3:143-155.

9. Moro CF, Yonekura M, Agrawal GK. Lotus-a source of food and medicine: current status and future perspectives in context of the seed proteomics. Int J Life Sci. 2013;7:1-5. Doi: $10.3126 /$ ijls.v7i1.6394.

10. Wu MJ, Wang L, Weng CY, Yen JH. Antioxidant activity of methanol extract of the lotus leaf. Am J Chin Med. 2003;31:687-698. doi: 10.1142/S0192415X03001429.

11. Saengkhae C, Arunnopparat W, Sungkhajorn P. Antioxidative activity of the leaf of Nelumbo nucifera Gaertn. on oxidative stress-induced erythrocyte hemolysis in hypertensive and normotensive rats. Thai J Physiol Sci. 2008;20:70-78.

12. Jung HA, Jung YJ, Yoon NY, Jeong DM, Bae HJ, Kim DW, et al. Inhibitory effects of Nelumbo nucifera leaves on rat lens aldose reductase, advanced glycation endproducts formation, and oxidative stress. Food Chem Toxicol. 2008;46:3818-26. Doi: 10.1016/j.fct.2008.10.004.

13. Huang B, He J, Ban X, Zeng H, Yao X, Wang Y. Antioxidant activity of bovine and porcine meat treated with extracts from edible lotus (Nelumbo nucifera) rhizome knot and leaf meat Science. 2001;87:46-53. doi: 10.1016/j. meatsci.2010.09.001.

14. Cour B, Molgaard P, Yi Z. Traditional Chinese medicine in treatment of hyperlipidaemia. J Ethnopharmacol. 1995;46:125-9. doi: 10.1016/0378-8741(95)01234-5.

15. Onishi E, Yamada K, Yamada T, Kaji K, Inoue H, Seyama Y, et al. Comparative effects of crude drugs on serum lipids. Chem Pharmaceut Bull. 1984;32:646-650. doi: 10.1248/ cpb.32.646.

16. Ono Y, Hattori E, Fukaya Y, Imai S, Ohizumi Y. Anti-obesity effect of Nelumbo nucifera leaves extract in mice and rats. J Ethnopharmacol. 2006;106:L238-244. doi: 10.1016/j. jep.2005.12.036.

17. Siegner R, Heuser S, Holtzmann U, Söhle J, Schepky A, Raschke T, et al. Lotus leaf extract and l-carnitine influence different processes during the adipocyte life cycle. Nutr Metab. 2010;7:66. doi: 10.1186/1743-7075-7-66.

18. Kashiwada Y, Aoshima A, Ikeshiro Y, Chen YP, Furukawa $\mathrm{H}$, Itoigawa $\mathrm{M}$, et al. Anti-hiv-benzylisoquinoline alkaloids and flavonoids from the leaves of Nelumbo nucifera, and structure-activity correlations with related alkaloids. Bioorgan Med Chem. 2005;13:1443-1448. doi: 10.1016/j. bmc.2004.10.020.

19. Ali L, Khan AK, Mamun MI, Mosihuzzaman M, Nahar N, Nur-e-Alam M, R, et al. Studies on hypoglycemic effects fruit pulp, seed and whole plant of Momordica charantia on normal and diabetic model rats. Planta Med. 1993;59:408412. doi: 10.1055/s-2006-959720.

20. Akhtar MS, Athar MA, Yaqub M. Effect of Momordica charantia on blood glucose levels of normal and alloxan diabetic rabbits. Planta Med. 1981;42:205-212. doi: 10.1055/ s-2007-971629. 